\title{
Overgrown grains appearing during sub-solvus heat treatment in a polycrystalline $\boldsymbol{\gamma} \boldsymbol{\gamma}^{\prime}$ Nickel-based superalloy
}

\author{
Marie-Agathe CHARPAGNE ${ }^{1,2^{*}}$, Jean-Michel FRANCHET ${ }^{3}$, Nathalie BOZZOLO ${ }^{1}$ \\ ${ }^{1}$ MINES ParisTech, PSL - Research University, CEMEF - Centre de mise en forme des matériaux, CNRS UMR 7635, \\ CS 10207 rue Claude Daunesse 06904 Sophia Antipolis Cedex, France, email : ma.charpagne@gmail.com \\ ${ }^{2}$ Safran Aircraft Engines, 171 boulevard de Valmy - BP 3192702 Colombes Cedex \\ ${ }^{3}$ Safran SA, SafranTech - Materials \& Process Division, rue des Jeunes Bois - Châteaufort - CS 8011278772 , \\ Magny-Les-Hameaux
}

Keywords : recrystallization, polycrystalline Nickel based superalloys, excessive grain growth, EBSD.

\section{Fundings}

This research was supported by the ANRT (French National Agency for Research and Technology) and Safran Aircraft Engines.

\section{Highlights}

1) Grain overgrowth during annealing after deformation is a case of critical recrystallization involving a limited number of nuclei.

2) The difference in stored energy between the overgrown grains and the matrix is the key microstructural driving force.

3) The self-impingement of the overgrown grains is the main size-limiting phenomenon, before Zener-pinning by second-phase particles.

4) The relative magnitudes of the driving forces at the deformed state enable to predict the occurrence of this phenomenon.

\begin{abstract}
The present article focuses on establishing the nature of excessive grain growth in a new $\gamma-\gamma^{\prime}$ superalloy, by investigating the underlying driving forces. Excessive grain growth upon annealing after deformation is a common phenomenon among Nickel-based superalloys. It shares common features with abnormal grain growth, but is fundamentally different, since not driven by capillarity forces. It consists in the selective and exaggerated growth of some grains, leading to the formation of heterogeneous microstructures and dramatically decreased mechanical properties. The present study demonstrates that the stored energy in the deformed matrix is the key driving force for exaggerated grain growth. The self-impingement of those grains is the main phenomenon limiting their growth, as Zener pinning by second-phase particles plays a minor role in the process. The absence of stored energy in the overgrown grains, the kinetics of their development and the
\end{abstract}


existence of an incubation time before their appearance in the microstructures lead to the conclusion that excessive grain growth is a case of 'critical' static recrystallization, involving a limited amount of nuclei. By evaluating the magnitude of the driving forces in presence before annealing, the microstructure evolutions during further annealing can be predicted, independently from the processing conditions.

\section{Introduction}

Polycrystalline Nickel-based superalloys are used as structural materials for high-temperature applications under extreme mechanical solicitations. Controlling their microstructure is of prime interest for guarantying their high mechanical properties. For rotating parts such as turbine disks, a fine and homogeneous grain size distribution is required, in order to guarantee their good fatigue resistance [1-3]. Nickel-based superalloys are usually forged at high temperature; their microstructure evolves during forging operations, depending on the thermomechanical conditions [4-7]. In such alloys, the $\gamma^{\prime}$ phase is present under the form of precipitates of various sizes. Primary ones are spherical and multi-micrometric. They are formed at the early stages of processing of the alloy and aim at pinning the grain boundaries during forging operations, enabling to control the grain size. Intragranular hardening precipitates are smaller and usually coherent with their hosting grain. Forging operations are typically performed at temperatures at which the pinning particles are present in the microstructure but the hardening ones are dissolved. This allows for a decreased flow stress while maintaining a small grain size. Intragranular precipitates then form again during cooling after deformation, with a distribution that mainly depends on the cooling rate from high temperature. Optimizing their distribution enables to adjust the mechanical properties and is thus the purpose of dedicated solution and ageing treatments $[8,9]$. Before such precipitation treatment, a solution treatment is performed, at a temperature below the $\gamma^{\prime}$ solvus at which all hardening precipitates are dissolved into the matrix while primary ones remain. The temperature of this treatment $-\mathrm{T}_{\text {sol }}$ - is adjusted regarding to the solvus temperature and depends on the alloy itself. However, some unwanted exaggerated grain growth may occur during this solution treatment, as already reported in various Nickel-based superalloys [10-18]. This selective growth of some grains leads to heterogeneous microstructures, consisting of fine grains and overgrown grains which size can be up to 10 times greater than the average. Such microstructures do not match the requirements $[1,19,20]$ on mechanical properties and have to be avoided. This phenomenon has been reported under different terminologies as "Abnormal Grain Growth" (AGG), "Critical Grain Growth" (CGG) [18], "Inhomogeneous Grain Growth" (IGG) [21] in the literature. Numerous studies have been carried on, aiming at determining the influence of the deformation and/or treatment conditions in a given alloy. However, only few of them focus on the metallurgical mechanisms of grain overgrowth and the underlying driving forces. This paper aims at clarifying the metallurgical phenomena involved, based on quantitative estimations of the forces acting in the microstructures, independently from the processing conditions.

\section{Literature background: Excessive grain growth phenomena in Nickel-based superalloys}

Excessive grain growth has already been reported in many Nickel-based superalloys, having undergone an annealing treatment after deformation. This phenomenon basically takes place at low strain levels $(\varepsilon \sim 0.1)$. The typical average grain size profile along a strain gradient involves a stationary size at very low strain levels, then a peak at a critical strain $\varepsilon_{c}$, followed by a decrease until 
the average grain size reaches a steady-state value. Dahlen [15] reports that the peak corresponds to a bimodal grain size distribution composed by a matrix of very fine grains and few grains of a much larger size, of lower dislocation density. This profile was observed in many $\psi-\gamma^{\prime}$ alloys, either cast like Waspaloy, Inconel-550, Nimonic 80A [10] or powder metallurgy (PM) alloys: N18 [11], René 95 [12], René 88 DT [13,14], Astroloy [15]. Similar behavior has also been reported in the $\psi-\gamma^{\prime \prime}-\delta$ alloy Inconel 718 [17,22-24]. The exact strain range at which the overgrown grains appear depends on the alloy itself, as well as on the deformation path and annealing conditions. But in any case, the microstructures retrieve a relatively fine monomodal grain size distribution at higher strains.

In an aim to prevent this phenomenon, the critical forging conditions could simply be avoided $[14,25]$. Following this idea, many studies have aiming at determining the critical forging window of a given alloy and a given solution treatment. This case-by-case approach has to be repeated after each modification of any of the forging parameters and annealing conditions. However, most industrial processes generate some strain gradients within the pieces, in which the critical strain levels cannot be avoided. In this context, understanding the microstructural mechanisms and driving forces leading to the overgrowth of some grains is the key for preventing it. Three main hypotheses have been proposed in the literature:

1. A favored crystallographic orientation: in highly textured materials -in which most boundaries are Low Angle Grain Boundaries (LAGBs) - a boundary separating one of those grains from another grain of different orientation will have an associated mobility intrinsically higher than a LAGB. [26]

2. A low Zener pinning pressure: a Zener pressure that is globally or locally too low (low particle volume fraction for a high particle radius, or areas locally depleted of particles) can enable the growth of selected grains. $[27,28]$

3. A favorable distribution of stored energy (SE): during deformation, energy is stored in the microstructure as dislocations and provides the driving force for the nucleation and the growth of recrystallized grains during subsequent annealing. In dynamically recrystallized and weakly deformed materials, the distribution of the SE is heterogeneous. If the SE in the deformed grains is high enough, the grains of lowest stored energy can grow by consuming those of higher SE. The grains with low SE are either already present in the deformed microstructure or are either recrystallization nuclei that form during the thermal treatment. [23]

The forged Nickel based superalloys are generally weakly or even not textured [25]. Hypothesis 1 thus does not sound relevant. By looking into more details, several cases can be identified depending on the deformation temperature (room temperature or high temperature) and on the solution temperature (sub-solvus or super-solvus), in which hypotheses 2 and 3 may play a major role.

In the case of room temperature deformation followed by a supersolvus annealing, the proposed hypothesis is that of critical static recrystallization $[13,29]$. Below the critical strain $\varepsilon_{c}$, the amount of SE present in the microstructure is too low to lead to any nucleation event ; only recovery can actually occur, preserving the original grain size distribution. During recrystallization under those critical conditions, the size of recrystallized grains is limited by the density of recrystallized grains density (number of such grains per unit volume) [14]. Within the critical strain range, the SE distribution only enables the nucleation of a limited number of nuclei. By calculating the SE 
distribution in the deformed microstructures, between neighboring grains, Miller et al. [13] have highlighted that Strain Induced Boundary Migration (SIBM) is likely to occur in a restricted number of areas in the microstructure. SIBM can lead to nucleation of a new grain by a bulging process along a former grain boundary. Once formed, those nuclei are in a favorable environment to grow by consuming the SE of the deformed grains. Grain boundary motion is hindered - or significantly slowed down - when the overall stored energy has been consumed and capillarity remains the only driving force acting on grain boundaries. In other words, the growth of such grains slows down when they meet each other. In such context, a limited number of nuclei will lead to a large abnormal grain size upon annealing. With increasing strain above the critical value, the nucleation density increases, leading to a decrease in the final recrystallized grain size. This evolution is consistent with the typical behavior reported in the literature. The involved mechanism of grain boundary motion driven by SE consumption meets hypothesis 3 listed above.

If the thermal treatment after cold deformation is performed at a sub-solvus temperature, second phase particles should prevent exaggerated grain growth $[12,14,24]$. Dahlen et al. [15] have nevertheless observed this phenomenon in Astroloy, in the two-phase domain. They have concluded that the development of overgrown grains results from the contribution of two combined factors: a SE level and distribution that enables the nucleation of a limited number of nuclei, associated with a Zener pinning pressure that is low enough so that the motion of the recrystallization front is not hindered. In this case, the main reason why few grains overgrow is still related to hypothesis 3 , and not to hypothesis 2 even though second phase particles are present.

In case of hot deformation, the critical strain range varies depending on the deformation conditions: temperature $(T)$ and strain rate $(\dot{\varepsilon})[11,18,30]$. During and after high temperature deformation, dynamic and post-dynamic recrystallization both take place in the microstructure. According to Blankenship et al. [14], the tendencies established for the case of room temperature deformation may be extrapolated, by taking into account dynamic phenomena. Most studies focus on the variation of the critical strain range, but the mechanisms of their formation and the driving forces triggering this phenomenon remain unclear [18]. Agnoli et al. [23] have proposed a grain to grain heterogeneous distribution of the SE in the deformed state, as the metallurgical origin of grain overgrowth, despite the presence of second phase particles. The grains that grow during the solution treatment are those of lowest SE. The question of their nucleation or not during treatment has not been solved yet.

Considering the literature background on the topic, it thus turns out that, among the three abovementioned, hypothesis 3 seems to be the most relevant. In this context, the aims of the present article are:

* To identify the characteristics of such grains and the conditions of their formation

* To highlight and discuss the metallurgical parameters triggering excessive grain growth, independently from the deformation conditions, by a means of a quantitative analysis of the driving and pinning forces in presence, with a special emphasis on stored energy 


\section{Experimental methods}

\subsection{Material and thermomechanical path}

The material selected for this study is the Rene 65 superalloy. Its chemical composition in weight percent is $13 \mathrm{Cr}-16 \mathrm{Co}-3.7 \mathrm{Ti}-2.1 \mathrm{Al}-4 \mathrm{Mo}-4 \mathrm{~W}-1 \mathrm{Fe}-0.7 \mathrm{Nb}-0.05 \mathrm{Zr}-0.016 \mathrm{~B}-$ bal Ni [31]. It is composed by a gamma matrix, which is a Nickel solid solution, mostly strengthened by Chromium, Cobalt and Molybdenum elements. The gamma prime phase is a $\mathrm{LI}_{2}$ ordered $\mathrm{Ni}_{3}(\mathrm{Al}, \mathrm{Ti})$, that has the shape of precipitates. The larger precipitates, which diameter vary from 1 to 5 micrometers, aim at pinning grain boundaries. Intragranular precipitates may also be present in the microstructures, but are typically dissolved back in solid-solution at forging temperatures. The samples considered in this paper have undergone:

i. Deformation at high temperature (either torsion or compression) in the sub-solvus domain, at temperatures from $900^{\circ} \mathrm{C}$ to $1090^{\circ} \mathrm{C}$ and strain rates from $0.001 \mathrm{~s}^{-1}$ to $0.1 \mathrm{~s}^{-1}$. Either air cooling or water quenching has followed deformation. The geometry of the double-cones is described in [32].

ii. A standard solution treatment, at the standard solution temperature, which will be referred to as $T_{\text {sol }}$. This temperature is below the solvus of the gamma prime phase, which is $1111^{\circ} \mathrm{C}$ [33]. The duration of the treatment is varied from 10 to 60 minutes. The samples are water quenched following that treatment.

Table 1 shows the chemical composition of $\gamma$ and $\gamma^{\prime}$ phases in a sample heat-treated at $T_{\text {sol }}$ for one hour then quenched in water, measured by Energy Dispersive X-Ray Spectroscopy (EDS) in a Scanning Electron Microscope (SEM).

\begin{tabular}{|c|c|c|c|c|c|c|c|c|c|}
\hline $\begin{array}{c}\text { Element } \\
\text { (wt\%) }\end{array}$ & Al & Co & Cr & Fe & Mo & Nb & Ni & Ti & W \\
\hline$\gamma^{\prime}$ precipitates & 4.3 & 7.6 & 3.1 & 0.3 & 0.6 & 1.0 & 70.8 & 10.8 & 1.5 \\
\hline$\gamma$ matrix & 1.8 & 14.3 & 17.9 & 1.0 & 3.7 & 0.8 & 53.7 & 3.3 & 3.5 \\
\hline
\end{tabular}

Table 1: Chemical composition of $\gamma$ and $\gamma^{\prime}$ phases at the solution treatment temperature (as measured by EDS on a sample heat-treated at $T_{\text {sol }}$ then quenched in water).

\subsection{Sample preparation and characterization}

All samples have been prepared for SEM and Electron Backscatter Diffraction (EBSD) characterizations. The polishing process involved the use of diamond suspensions followed by vibratory polishing with a $0.05 \mu \mathrm{m}$ colloidal silica suspension.

* Driving forces in presence in the deformed state

The distribution of primary $\gamma^{\prime}$ precipitates was assessed by image processing on backscattered electrons images, using the Visilog ${ }^{\circledR}$ software as described in [32]. The total volume fraction of particles $f$ and their average equivalent-circle diameter $d$ have been measured. Over 4,000 precipitates were analyzed for each microstructure. The magnitude of the Zener pressure $P_{Z}$ 
associated to those particles has been calculated using the formula (1), where $f_{G B}$ is the fraction of particles located on grain boundaries, $d_{G B}$ is their average equivalent-circle diameter and $\gamma$ is the grain boundary energy $\left(0.8 \mathrm{~J} / \mathrm{m}^{2}\right.$ for a similar Nickel based superalloy at $1000^{\circ} \mathrm{C}$ [34]). Since most precipitates are located on the grain boundaries at this state, the assumptions $f=f_{G B}$ and $d=$ $d_{G B}$ were made.

$$
P_{Z}=\frac{3 \gamma f_{G B}}{d_{G B}}
$$

Assuming that all grain boundaries get pinned by the $\gamma^{\prime}$ particles, one can define the Zener limited grain size, which is defined (originally for capillarity driven grain boundary motion) by the formula (2):

$$
D_{Z}=\frac{3 d_{G B}}{4 f_{G B}}(2)
$$

The deformed microstructures have been mapped by means of a coupled EDS/EBSD technique described in [35], using a Bruker system mounted on a Zeiss Supra 40 Field-Emission Gun microscope operated at $15 \mathrm{kV}$. The EDS/EBSD data has been processed with the Bruker Esprit software package for discriminating $\gamma$ and $\gamma^{\prime}$ phases. The crystal orientation maps were then post-processed using the TSL OIM 5.0 software. A misorientation angle threshold of $15^{\circ}$ was used for defining a grain boundary. Twin boundaries are defined as $60^{\circ}$ rotations around $\langle 111\rangle$ directions, with a tolerance of $5^{\circ}$. Twin boundaries are considered as internal crystal defects in grain size calculations.

The difference in stored energy $P_{S E}$ between the deformed (def) and recrystallized (reX) grains is calculated using formula (3):

$$
P_{S E}=0.5\left(\bar{\rho}_{\text {tot }}^{\text {def }}-\bar{\rho}_{\text {tot }}^{r e X}\right) G b^{2}(3)
$$

where $G$ is the shear modulus of the alloy (14.77 GPa) [33] and $b$ is the magnitude of the Bürgers vector $(0.25 \mathrm{~nm})$. The average total dislocation density of a population $i, \bar{\rho}_{\text {tot }}^{i}$, is the average value of the total dislocation density of all the grains belonging to this population (deformed or recrystallized). Each grain of the microstructure has a total dislocation density $\rho_{\text {tot }}$ that is the sum of the contributions of Geometrically Necessary Dislocations (GNDs) and Statistically Stored Dislocations (SSDs).

$$
\rho_{t o t}=\rho_{G N D s}+\rho_{S S D S}
$$

In a rough approximation, the density of GNDs can be correlated to the intragranular misorientations measured on EBSD maps, by using the formula (5) [36].

$$
\rho_{G N D S}=\frac{K}{b} \frac{\theta}{x}(5)
$$

$b$ is the norm of the Bürgers vector. $\theta$ is the misorientation angle between two points in the grain and $x$ is the distance between those points. Instead of using individual $\theta$ values evaluated from single pairs of points, one can replace it by the Kernel Average Misorientation (KAM) parameter. The KAM value is the average value of the misorientation angles $\theta$ between a given point and all its neighbours located within a given distance. Using the Grain Average Misorientation (GAM) parameter instead of the KAM is a better choice in the present work. It enables to obtain an estimation of the GND density at the scale of the grain. The GAM value is the average value of the misorientation angles $\theta$ between 
all pairs of neighbouring points in a grain. The corresponding dislocation density, $\rho_{G N D S}^{G A M}$, can then be estimated in each grain by using the GAM value and $x$ being the measurement step size. This leads to formula (6):

$$
\rho_{G N D S}^{G A M}=\frac{K}{b} \frac{G A M}{x}(6)
$$

where $b$ is the norm of the Bürgers vector and $K$ is a parameter which depends on the hypothesis made on the dislocation structure. $K=3$ was used in the present work [37]. As a rough estimation, the density of GNDs can be considered as a fraction of the total dislocation density [38] :

$$
\rho_{\text {tot }}=c * \rho_{G N D S}(7)
$$

where $\mathrm{c}$ is a constant value greater than 1 . Its value varies from one grain to another, depending on the crystal orientation and the plastic strain, but is actually very difficult to be determined. Instead of making a poorly justified assumption on this parameter value, the choice was made to consider only GNDs, so the assumption $c=1$ was made. One must thus keep in mind that the evaluated stored energy values are very likely to be underestimated.

In each microstructure, the distribution of the GAM values was analysed and a threshold value has been determined, which enables to split the grains into deformed and recrystallized populations. Two partitions were created using the TSL OIM software, enabling to calculate the $\bar{\rho}_{\text {tot }}^{\text {def }}$ and $\bar{\rho}_{\text {tot }}^{\text {reX }}$ quantities in formula (3).The obtained values of SE are biased by the noise associated to the EBSD technique itself. Therefore, a normalized, unitless value has been used. This value, $P_{S E}^{\prime}(8)$, is the ratio of the actual value of $P_{S E}$ in the microstructure, divided by the maximum value of $P_{S E}$ measured among all the studied microstructures, $P_{S E}^{\max }$. It will be referred to as the normalized stored energy parameter in the following.

$$
P_{S E}^{\prime}=\frac{P_{S E}}{P_{S E}^{\max }}(8)
$$

\section{* Grain size measurements in the solution treated samples}

The equivalent circle diameter of the largest grain $D_{M A X}$ has been determined and the average grain size $\bar{D}$ has been measured on optical micrographs using the intersection method. The grain boundaries of the solution treated samples have been revealed using the Kalling etchant. 


\section{Results}

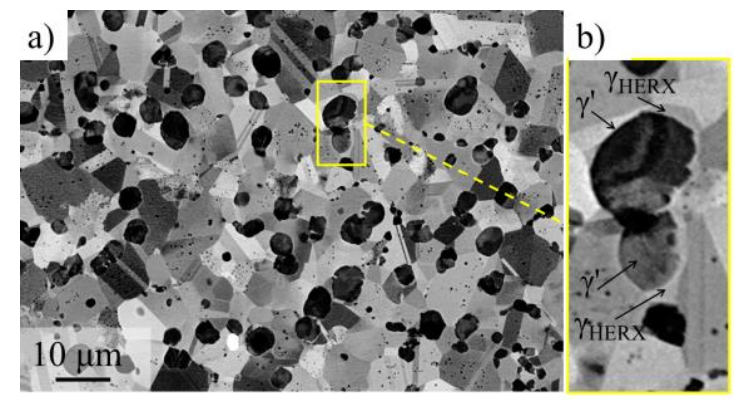

Figure 1: Back-scattered electrons image of the microstructure deformed at $1040^{\circ} \mathrm{C}$ to a strain of 0.15 at a strain rate of $0.01 \mathrm{~s}^{-1}$ and water quenched. a) Overview. b) Zoom onto primary $\gamma^{\prime}$ precipitates with HERX grains.

Figure 1a shows the microstructure of the sample deformed at $1040^{\circ} \mathrm{C}$ and $0.01 \mathrm{~s}^{-1}$. This microstructure mostly consists of deformed grains. Primary $\gamma^{\prime}$ precipitates are located on grain boundaries. Few grains have recrystallized by the hetero-epitaxial (HERX) mechanism $[32,39]$. They represent $8 \%$ of the surface fraction of all matrix grains. Two typical examples are highlighted on Figure 1b. Conventional dynamic recrystallization has not been triggered in the present microstructure.

\subsection{Metallurgical characteristics of overgrown grains}

Figure 2 displays a set of EDS/EBSD maps acquired on the sample of Figure 1 after one hour exposure at $T_{\text {sol }}$. Two overgrown grains are present in the area of interest and are numbered on the figure. They are about ten times larger than matrix grains. Figs. $2 a$ and $2 b$ reveal that such grains contain multiple annealing twin boundaries. Overgrown grains also contain many intragranular and incoherent primary $\gamma^{\prime}$ precipitates. It should be noted that no difference in either shape or size distribution of primary $\gamma^{\prime}$ precipitates was found between the zones in which excessive growth had occurred. This fact discards the hypothesis 2 mentioned in the literature section. It also shows that the Zener-pressure was not sufficient to pin the grain boundaries of those specific grains. Fig. $2 \mathrm{c}$ shows that the Grain Orientation Spread (GOS) of the overgrown grains are below $0.5^{\circ}$, which is about the angular resolution achievable by EBSD under conventional mapping settings. On the opposite, small grains are pinned by $\gamma^{\prime}$ precipitates. Their average GOS parameter is $0.7^{\circ}$, which indicates that they contain some SE. The very low SE level of the overgrown grains suggests that they have developed by consuming the SE of the smaller ones. The same observation has previously been reported for the Inconel 718 alloy [23]. 
a)
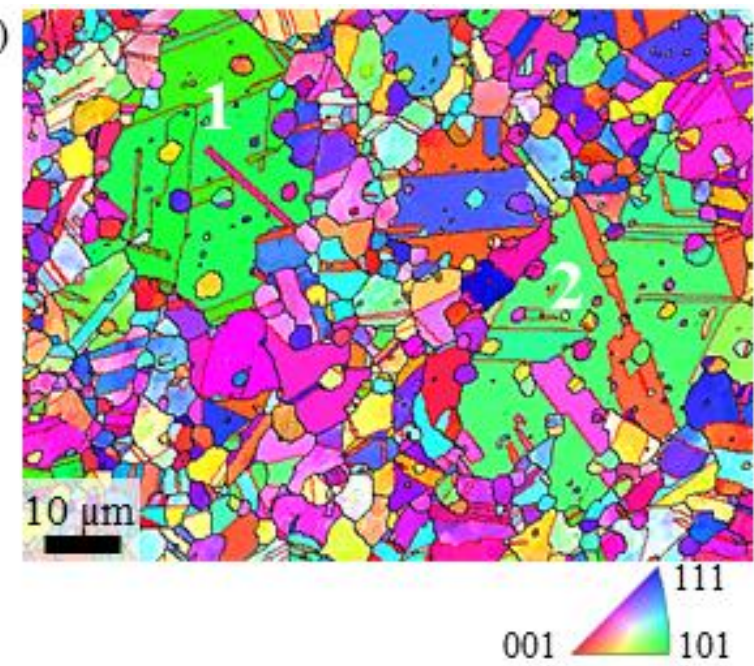

b)

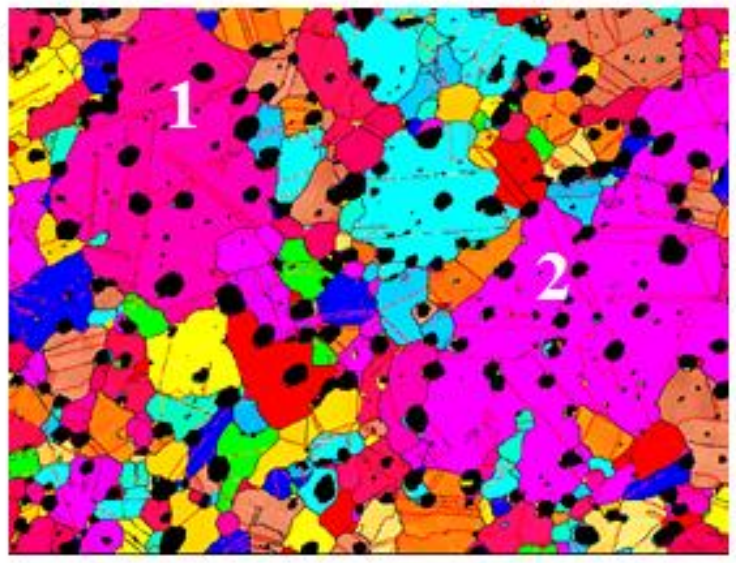

c)

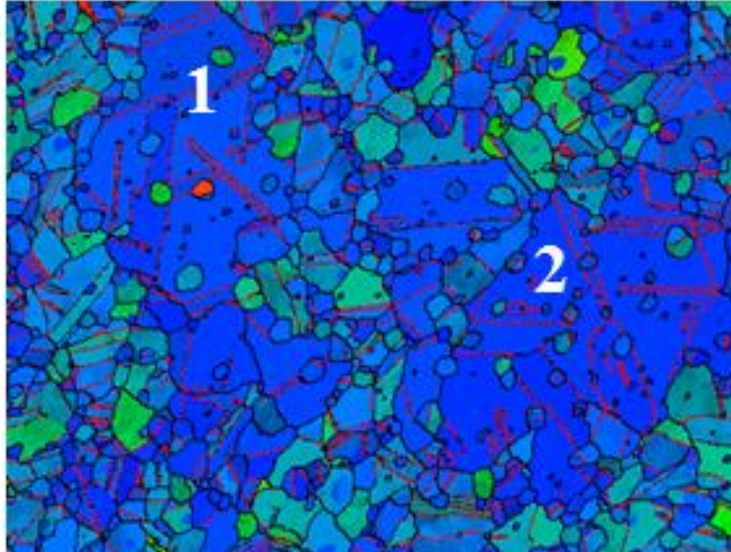

0

$3^{\circ}$

Figure 2: EDS-EBSD maps of a sample deformed at $1040^{\circ} \mathrm{C}, \dot{\varepsilon}=0.01 \mathrm{~s}-1$ and $\varepsilon=0.15$, after 30 minutes of solution treatment. a) Orientation map within a color code indicating which crystallographic direction is parallel to the normal to the scanned section; b) $v^{\prime}$ phase (identified by EDS) in dark and $v$ grains colored arbitrarily, ignoring twin boundaries; c) Grain Orientation Spread (GOS) map. All maps are superimposed with the Index of Quality map; grain boundaries (misorientation angles $>15^{\circ}$ ) are represented in black and annealing twins in red. (For interpretation of the references to color in this figure legend, the reader is referred to the web version of this article). 


\subsection{Microstructure development in a strain gradient}

Figure 3 shows the microstructure development in the strain gradient of the sample deformed at $1040^{\circ} \mathrm{C}, 0.001 \mathrm{~s}^{-1}$ after 0.5 hour exposure at $T_{\text {sol }}$. The same two populations of grains can be identified on the sequence: fine dynamically recrystallized grains and overgrown grains. Overgrown grains appear progressively in the microstructure. The first initial grain is arrowed on the figure. As the strain increases, they become more numerous and smaller. At the strain level of $\varepsilon=0.17$, they have fully invaded the microstructure (dashed line on Fig. 3). Starting from $\varepsilon=0.15$, their grain boundaries are not pinned by the $\gamma^{\prime}$ precipitates anymore. With increasing strain, those grains become more numerous and their size seems to be controlled by their number density rather than by the presence of primary $\gamma^{\prime}$ precipitates. The relative influence of the driving and pinning forces in presence will be discussed in paragraph 5.2.

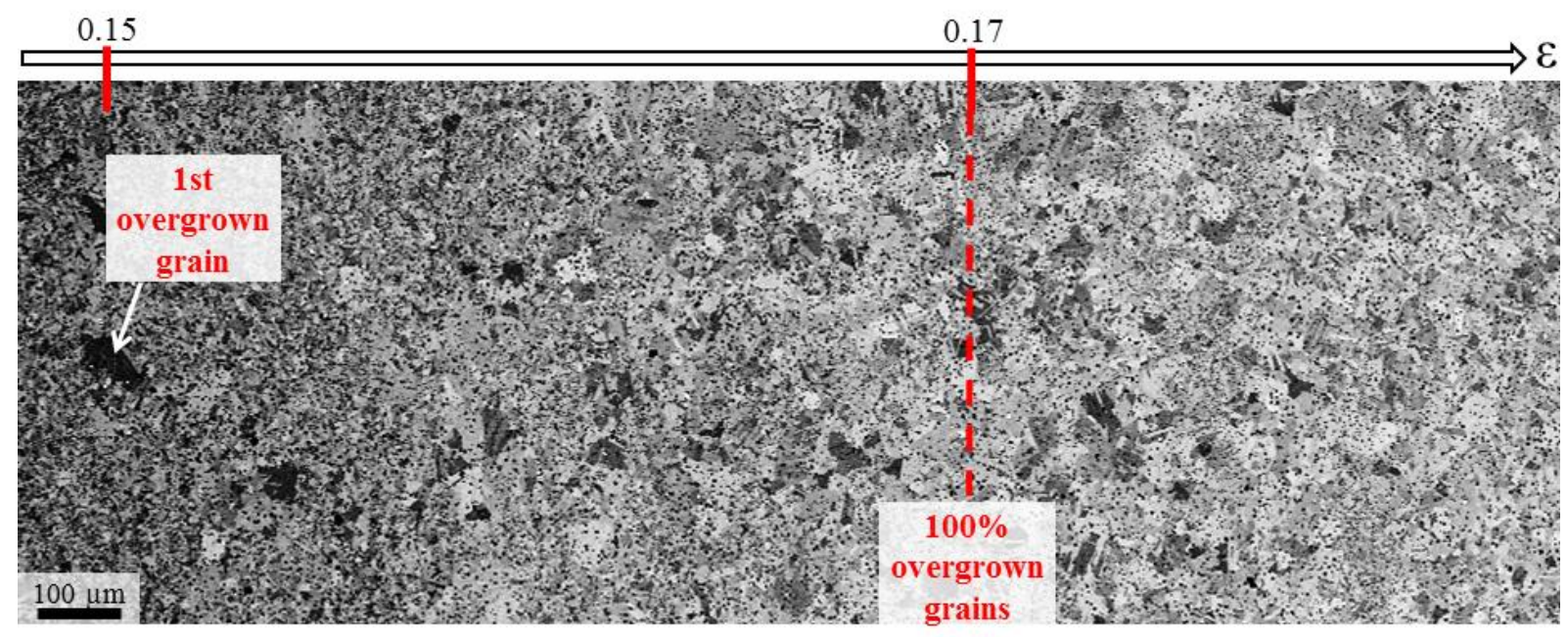

Figure 3: Back-scattered electron image showing the microstructure development along the strain gradient of a sample deformed at $1040^{\circ} \mathrm{C}$ and $0.001 \mathrm{~s}^{-1}$ and solution-treated for 30 minutes at $T_{S o l}$.

The same behavior has been obtained after varying the strain rate and/or the solution treatment duration. Figure 4 presents the evolution of the average and maximum grain size in a sample deformed at $1040^{\circ} \mathrm{C}$ and $\dot{\varepsilon}=0.01 \mathrm{~s}^{-1}$ and heat-treated for one hour at $T_{\text {sol }}$. The $D_{\text {MAX }}$ profile corresponds to the trends previously reported in the literature. From $\varepsilon=0$ to $\varepsilon^{A}=0.04$, the microstructure remains fine and homogeneous, and the grains are small and deformed. At $\varepsilon^{A}$, the first overgrown grain appears. As the strain increases from $\varepsilon^{A}$ to $\varepsilon^{B}=0.12$, more and more overgrown grains are visible, leading to an increase of the average grain size. However the size of the overgrown grains decreases as the strain increases. In this strain range, the grain size distribution is clearly bimodal. From $\varepsilon^{B}$ to $\varepsilon^{C} \leq 0.25$, the microstructure is solely composed by overgrown grains, which size further decreases and number density further increases. The grain size distribution becomes homogeneous again. At $\varepsilon>\varepsilon^{C}$, the average grain size becomes small back again. 


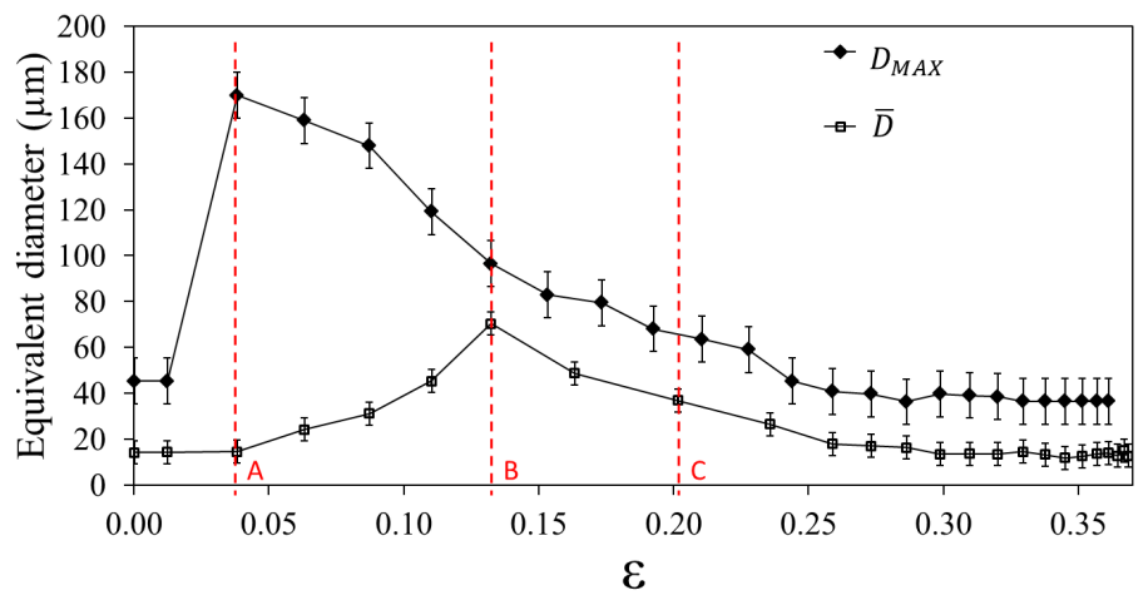

Figure 4: Evolution of the maximum and average grain sizes along the strain gradient of a sample deformed at $1040^{\circ} \mathrm{C}, \dot{\varepsilon}=0.01 \mathrm{~s}^{-1}$ and solution-treated for one hour. Landmark $\varepsilon^{A}$ : first overgrown grain; Landmark $\varepsilon^{B}$ : microstructure fully replaced by overgrown grains; Landmark $\varepsilon^{C}$ : fine and homogeneous microstructure.

\subsection{Microstructure development as a function of time exposure at $T_{\text {sol }}$}

The same sample presented on Figure 1 has been treated for various annealing times $t$ at $T_{\text {sol }}$. The critical annealing strains $\varepsilon^{A}(t)$ and the corresponding maximum grain diameters $D_{M A X}^{A}(t)$ have been measured and reported in Table 2. As a function of time, the critical strain at which the first grain appears decreases, and the associated maximum diameter increases. This can be interpreted in terms of incubation time. The overgrown grains appear after an incubation time which increases as strain decreases (for the present deformation conditions: 10 minutes for a strain of $0.19,20$ minutes for a strain of 0.17 , etc.). At the critical strain $\varepsilon^{A}(t)$, grain overgrowth becomes more and more pronounced as strain decreases.

\begin{tabular}{|c|c|c|c|c|c|c|}
\hline$t(\mathbf{m i n})$ & $\mathbf{0}$ & $\mathbf{1 0}$ & $\mathbf{2 0}$ & $\mathbf{3 0}$ & $\mathbf{4 0}$ & $\mathbf{6 0}$ \\
\hline$\varepsilon_{c}^{t}$ & No AGG & 0.19 & 0.17 & 0.15 & 0.14 & 0.05 \\
\hline$D_{M A X}^{t}(\boldsymbol{\mu m})$ & No AGG & 40 & 55 & 70 & 75 & 170 \\
\hline
\end{tabular}

Table 2: Critical strain to onset grain overgrowth and maximum grain size at the critical strain, obtained in the sample deformed at $1040^{\circ} \mathrm{C}, 0.01 \mathrm{~s}^{-1}$, after various time exposure at $T_{\text {sol }}$.

\section{Discussion}

From the results presented previously, grain overgrowth is triggered during the solution treatment, when the deformation is above a critical strain $\varepsilon^{A}$. The profile of $D_{\text {MAX }}$ obtained on Fig. 4 is consistent with those reported in previous studies on similar phenomena $[13,15,18,23]$. $\varepsilon^{A}$ varies with the deformation conditions and the time exposure at $T_{\text {sol }}$. The overgrown grains are free of SE and develop in a deformed matrix. 


\subsection{Stored energy as the key driving force for grain overgrowth}

The stored energy has been estimated from EBSD data using equations 3 to 8 . Figure 5 shows the variation of $P_{S E}$ as a function of strain, in a sample deformed at $1040^{\circ} \mathrm{C}$ and $0.01 \mathrm{~s}^{-1}$. The relation between $P_{S E}$ and the strain is linear. Since recrystallization has not occurred yet in that domain, the results presented previously can be similarly interpreted as a function of strain or SE. In other words, the principle "the higher the strain, the higher the stored energy" will be assumed.

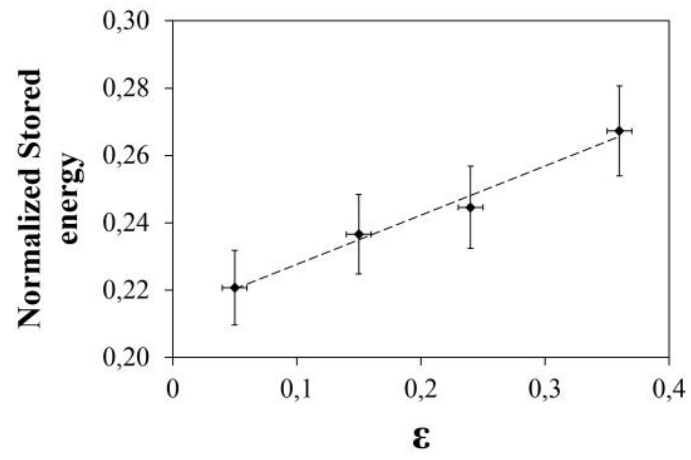

Figure 5: Normalized stored energy parameter as a function of strain in the sample deformed at $1040^{\circ} \mathrm{C}$ and $0.01 \mathrm{~s}^{-1}$.

a)

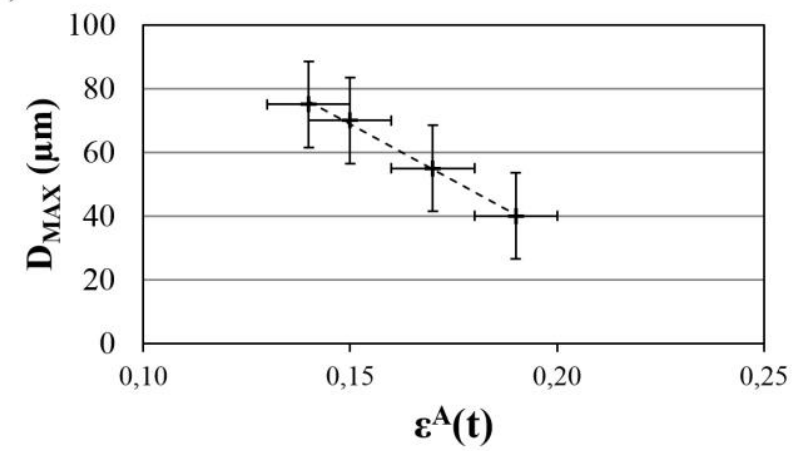

b)

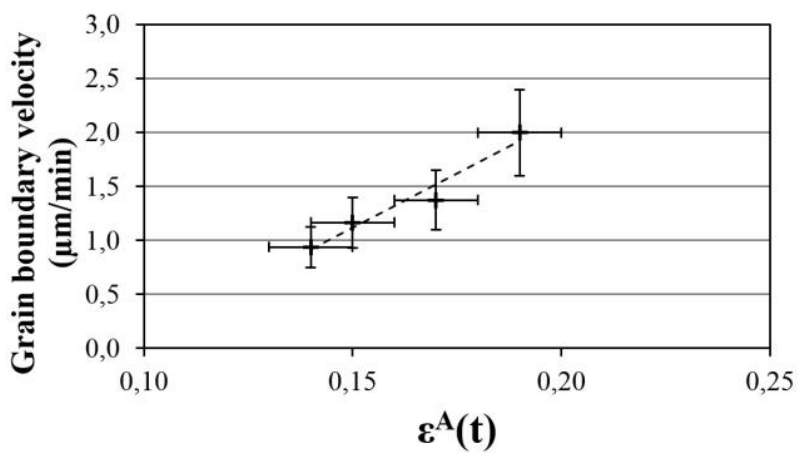

Figure 6: Kinetics of development of the overgrown grains in the sample deformed at $1040^{\circ} \mathrm{C}$ and $0.01 \mathrm{~s}-1$. a) Maximum grain diameter as a function of the corresponding strain. b) Grain boundary velocity as a function of strain, for different annealing times.

Figure 6 has been obtained from the results presented in Table 2. Figure 6-a shows the dependence of the size of the largest grain on the critical strain level. The relation is quasi linear. According to Table 2, they are actually both proportional to the annealing time. The grain boundary velocity of the 
overgrown grains $(V)$ can be estimated using the formula $V(t)=0.5 * \frac{D_{M A X}(t)}{t}$. From Figure $6-\mathrm{b}$, the grain boundary velocity increases linearly with the critical strain level for annealing times from 10 to 40 minutes. Using Fig. 5 again, the grain boundary velocity is proportional to the SE. This result is in accordance with the equation $V=M P$, in which $\mathrm{M}$ is the mobility of the grain boundary and $\mathrm{P}$ is the driving force for grain boundary motion, here the SE.

This tendency shows that the SE plays a major role in the development of overgrown grains in the considered sample where the Zener pressure is constant.

\subsection{Balance of forces controlling grain overgrowth}

The SE present in the matrix is the key driving force allowing grain overgrowth, despite the presence of primary $\gamma^{\prime}$ precipitates, which oppose a Zener pressure to the grain boundary motion. Indeed, from Figure 3, once the grain size distribution becomes homogeneous again, the grains seem to be pinned by second-phase particles. However, the real influence of second phase particles on the whole microstructure evolution sequence remains to be clarified.

Figure 7 aims at correlating the occurrence (color) and magnitude (symbol size) of grain overgrowth during the solution treatment to the Zener pinning pressure (X-axis) and to the energy stored in the initial deformed state ( $\mathrm{Y}$-axis). This plot gathers the results obtained on over 50 samples deformed and cooled under various conditions, then submitted to an hour exposure at $T_{\text {sol }}$. Red markers correspond to microstructures in which grain overgrowth has occurred during the solution treatment. Green markers correspond to homogeneous grain size distributions with grain boundaries pinned by particles. The size of the markers is related to the size of the largest grain observed after the solution treatment, following the scale shown at the bottom of the figure.

The typical microstructure evolutions for a constant Zener pinning pressure and in a gradient of stored energy is highlighted by the arrow " $A$ ". The typical microstructure behavior described in section 4 is retrieved. At low SE levels, the microstructure remains stable. As the SE increases, grain overgrowth is triggered. It starts with very large grains, which size then diminishes until a fine and homogeneous microstructure is obtained.

With varying the Zener pinning pressure, five domains can be depicted on this figure. The first domain corresponds to the microstructures in which the SE was below the detection limit of the EBSD technique and the Zener pinning pressure is moderate (about $80 \mathrm{kPa}$ ). In such microstructures, a grain that is free of SE will be surrounded by similar grains. The driving force for its growth is thus very low and the maximum grain size remains of the order of magnitude of the Zener limit (Table 2). The second domain corresponds to microstructures having undergone deformation at high temperature $\left(1080^{\circ} \mathrm{C}-1090^{\circ} \mathrm{C}\right)$, close to the $\gamma^{\prime}$ phase solvus temperature. The partial dissolution of the $\gamma^{\prime}$ precipitates at such temperatures leads to a decrease of the associated Zener pressure, down to $50-60 \mathrm{kPa}$. The SE present in the microstructure has enabled the growth of some grains, which has nevertheless been limited by the Zener pressure. The resulting microstructures are thus homogeneous with a large average grain size. The third zone corresponds to samples forged at low to medium temperatures $\left(950-1040^{\circ} \mathrm{C}\right)$ and high strain levels $(\varepsilon>0.6)$. In such microstructures, both the Zener pinning pressure and the SE in the deformed part of the microstructure are high. This leads to full recrystallization during the solution treatment and a fine and homogeneous microstructure with grain boundaries efficiently pinned by primary $\gamma^{\prime}$ precipitates. The fourth domain 
is that of grain overgrowth. It corresponds to similar configurations in terms of Zener pinning pressure but the microstructures deformed at lower strain levels. In such cases, the solution treatment leads to the formation of only few grains of low SE, which can overgrow. The fifth domain corresponds to microstructures having undergone deformation at high temperatures $\left(1040^{\circ} \mathrm{C}-\right.$ $1070^{\circ} \mathrm{C}$ ) and high strain levels, leading to both medium Zener pinning pressure and SE. In this case, the $\mathrm{SE}$ is obviously high enough to promote the formation of many recrystallized grains during the solution treatment which impinge on each other and thus keep reasonable sizes, below the Zener limit.

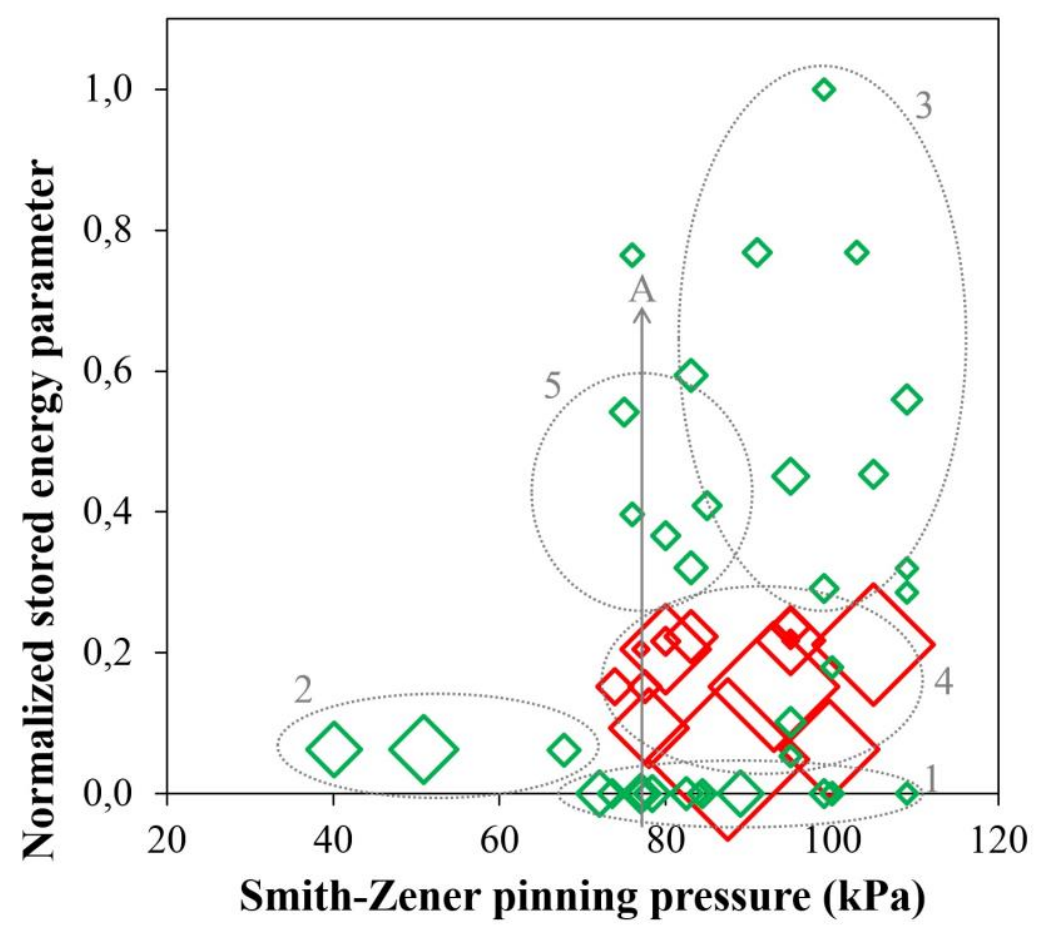

Figure 7: Influence of the stored energy of the deformed grains and of the Zener pinning pressure, on the occurrence of grain overgrowth, during the solution treatment.

\begin{tabular}{|c|c|c|c|c|c|}
\hline Domain & $\mathbf{1}$ & $\mathbf{2}$ & $\mathbf{3}$ & $\mathbf{4}$ & $\mathbf{5}$ \\
\hline$D_{M A X}(\mu m)$ & $30-40$ & $30-60$ & 20 & $80-200$ & $25-30$ \\
\hline$D_{Z}(\mu m)$ & $25-30$ & $30-40$ & 20 & 25 & $25-30$ \\
\hline
\end{tabular}

Table 3: Comparison of the size of the largest grain with the Zener limited grain size, estimated assuming only the contribution of capillarity to the driving force. 


\subsection{On grain overgrowth as a particular case of static recrystallization}

The presence of HERX grains with a low stored energy generates a heterogeneous distribution of SE in the microstructure. Yet, Agnoli et al. [23] have identified such heterogeneities as a key factor for the trigger of grain overgrowth, in Inconel 718. Following this hypothesis, the few growing grains would be the least deformed in the deformed microstructure, or they could result from a nucleation process occurring during the solution treatment. In case of a $\psi-\gamma^{\prime}$ microstructure with HERX grains, an additional hypothesis should be introduced with regards to the origin of the overgrown grains: they could potentially result from the growth of HERX grains. This will be investigated in details in a dedicated study. The present discussion is focused on the reason why those grains can grow to such extent, rather than on their exact origin. However, two features can already lead to think that overgrown grains are not a result of the growth of HERX nuclei. First, HERX is restricted to $\gamma-\gamma^{\prime}$ alloys and very similar grain overgrowth has been reported in $\psi-\gamma^{\prime \prime}$ alloys such as Inconel 718 as well. Second, the data presented in Table 2 shows the existence of an incubation time before the appearance of overgrown grains. If the HERX nuclei were to grow abnormally in the microstructure, then no incubation time would be observed, since they are already present at the deformed stage. The same reasoning holds for the growth of low SE grains that was suggested by Agnoli et al.

On the other hand, the incubation time is shorter at higher strains and increases as strain decreases, which is a very typical behavior in a static recrystallization process. This is a strong argument in favor of the hypothesis that overgrown grains result from a conventional static recrystallization mechanism, following the occurrence of nucleation events during the solution treatment. In addition, the superimposed profiles of $D_{M A X}$ and $\bar{D}$ displayed on Fig. 4 are also consistent with a static recrystallization phenomenon.

\section{Conclusions}

- Grain overgrowth occurring during solution treatment after hot deformation is most likely a case of critical static recrystallization.

- The occurrence of nucleation events as well as the actual mechanism of recrystallization leading to overgrown grains remains to be investigated in more details. However, many features reinforce the hypothesis of the effective occurrence of nucleation events during the solution treatment.

- The difference in stored energy between the deformed and non-deformed grains is clearly the key factor promoting the growth of the nucleated grains during the solution treatment.

- Their size is limited by both recrystallized grains impingement (nucleation density effect), then possibly by Zener pinning as well. The Zener pinning pressure exerted by primary $\gamma^{\prime}$ precipitates is nevertheless easily overcome by the difference in stored energy.

\section{References}

[1] J. Miao, T.M. Pollock, J. Wayne Jones, Microstructural extremes and the transition from fatigue crack initiation to small crack growth in a polycrystalline nickel-base superalloy, Acta Mater. 60 (2012) 2840-2854. doi:10.1016/j.actamat.2012.01.049. 
[2] J.C. Healy, L. Grabowski, C.J. Beevers, Monitoring Fatigue of a Nickel-base Superalloy at Positive and Negative Stress Ratios using an Optical System, Fatigue Fract. Eng. Mater. Struct. 15 (1992) 309-321. doi:10.1111/j.1460-2695.1992.tb01272.x.

[3] M.L. Brogdon, A.H. Rosenberger, Evaluation of the influence of grain structure on the fatigue variability of Waspaloy, in: Superalloys 2008, 11th Int. Symp. Superalloys proceedings, (2008) 583-588.

[4] H. Jiang, L. Yang, J. Dong, M. Zhang, Z. Yao, The recrystallization model and microstructure prediction of alloy 690 during hot deformation, Mater. Des. 104 (2016) 162-173. doi:10.1016/j.matdes.2016.05.033.

[5] Y. Liu, Y. Ning, X. Yang, Z. Yao, H. Guo, Effect of temperature and strain rate on the workability of FGH4096 superalloy in hot deformation, Mater. Des. 95 (2016) 669-676. doi:10.1016/j.matdes.2016.01.032.

[6] Y.C. Lin, D.-G. He, M.-S. Chen, X.-M. Chen, C.-Y. Zhao, X. Ma, Z.-L. Long, EBSD analysis of evolution of dynamic recrystallization grains and $\delta$ phase in a nickel-based superalloy during hot compressive deformation, Mater. Des. 97 (2016) 13-24. doi:10.1016/j.matdes.2016.02.052.

[7] L. Tan, Z. Huang, F. Liu, G. He, X. Wang, L. Huang, Y. Zhang, L. Jiang, Effects of strain amount and strain rate on grain structure of a novel high Co nickel-based polycrystalline superalloy, Mater. Des. 131 (2017) 60-68. doi:10.1016/J.MATDES.2017.06.004.

[8] A. Mostafaei, Y. Behnamian, Y.L. Krimer, E.L. Stevens, J.L. Luo, M. Chmielus, Effect of solutionizing and aging on the microstructure and mechanical properties of powder bed binder jet printed nickel-based superalloy 625, Mater. Des. 111 (2016) 482-491. doi:10.1016/j.matdes.2016.08.083.

[9] H. Wu, J. Li, F. Liu, L. Huang, X. Zeng, Q. Fang, Z. Huang, L. Jiang, A high-throughput methodology search for the optimum cooling rate in an advanced polycrystalline nickel base superalloy, Mater. Des. 128 (2017) 176-181. doi:10.1016/J.MATDES.2017.05.025.

[10] R.F. Decker, A.I. Rush, A.G. Dano, J.W. Freeman, Abnormal Grain Growth in Nickel-based heatresistant alloys, in: Natl. Advis. Com. Aeronaut. Tech. Note 4082, National Advisory Commitee for Aeronautics, (1957) p. 1.

[11] M. Soucail, M. Marty, H. Octor, Development of coarse grain structures in a powder metallurgy nickel base superalloy N18, Scr. Mater. 34 (1996) 519-525. doi:10.1016/13596462(95)00522-6.

[12] M.N. Menon, F.J. Gurney, Microstructural investigation of the growth of large grains in prealloyed powder extrusions of a nickel base superalloy, Metall. Trans. A. 7 (1976) 731-743. doi:10.1007/BF03186806.

[13] V.M. Miller, A.E. Johnson, C.J. Torbet, T.M. Pollock, Recrystallization and the Development of Abnormally Large Grains After Small Strain Deformation in a Polycrystalline Nickel-Based Superalloy, Metall. Mater. Trans. A. 47 (2016) 1566-1574. doi:10.1007/s11661-016-3329-6.

[14] C. Blankenship, M. Henry, J. Hyzak, R. Rohling, E. Hall, Hot-die forging of P/M Ni-base superalloys, in: Superalloys 1996, 8th Int. Symp. Superalloys, (1996) 653-662.

[15] M. Dahlen, L. Winberg, Grain coarsening of PM Nickel-base superalloy by critical strain annealing, Met. Sci. (1979) 163-169. 
[16] N. Bozzolo, A. Agnoli, N. Souaï, M. Bernacki, R.E. Logé, Strain Induced Abnormal Grain Growth in Nickel Base Superalloys, Mater. Sci. Forum. 753 (2013) 321-324.

doi:10.4028/www.scientific.net/MSF.753.321.

[17] A. Agnoli, M. Bernacki, R. Loge, J.-M. Franchet, J. Laigo, N. Bozzolo, Understanding and modeling of grain boundary pinning in Inconel 718, in: Superalloys 2012, 12th Int. Symp. Superalloys proceedings (2012) 73-82.

[18] E. Huron, S. Srivatsa, E. Raymond, Control of grain size via forging strain rate limits for R'88DT, in: Superalloys 2000, 9th Int. Symp. Superalloys proceedings (2000) 49-58.

[19] J. Miao, T.M. Pollock, J. Wayne Jones, Crystallographic fatigue crack initiation in nickel-based superalloy René 88DT at elevated temperature, Acta Mater. 57 (2009) 5964-5974. doi:10.1016/j.actamat.2009.08.022.

[20] A. Shyam, C. Torbet, S. Jha, J. Larsen, M. Caton, C. Szczepanski, T.M. Pollock, J. Jones, Development of ultrasonic fatigue for rapid, high temperature fatigue studies in turbine engine materials, in: Superalloys 2004, 10th Int. Symp. Superalloys proceedings (2004) 259268.

[21] I. M. D. Parr, T. JAckson, M. Hardy, C. Argyrakis, K. Severs, V. Saraf, J.M. Stumpf, Inhomogeneous Grain Coarsening Behavior in Supersolvus Heat Treated Nickel-Based Superalloy RR1000, in: Superalloys 2016, 13th Int. Symp. Superalloys proceedings (2016) 447456. doi:DOI: 10.1002/9781119075646.ch48.

[22] N. Bozzolo, S. Jacomet, R.E. Logé, Fast in-situ annealing stage coupled with EBSD: A suitable tool to observe quick recrystallization mechanisms, Mater. Charact. 70 (2012) 28-32. doi:10.1016/j.matchar.2012.04.020.

[23] A. Agnoli, M. Bernacki, R. Logé, J.-M. Franchet, J. Laigo, N. Bozzolo, Selective Growth of Low Stored Energy Grains During $\delta$ Sub-solvus Annealing in the Inconel 718 Nickel-Based Superalloy, Metall. Mater. Trans. A. 46 (2015) 4405-4421. doi:10.1007/s11661-015-3035-9.

[24] A. Agnoli, N. Bozzolo, R. Logé, J.-M. Franchet, J. Laigo, M. Bernacki, Development of a level set methodology to simulate grain growth in the presence of real secondary phase particles and stored energy - Application to a nickel-base superalloy, Comput. Mater. Sci. 89 (2014) 233241. doi:10.1016/j.commatsci.2014.03.054.

[25] D. Whitis, Recovery and recrystallization after critical strain in the nickel-based superalloy Rene 88DT Databases Full Record, in: Superalloys 2004, 10th Int. Symp. Superalloys, (2004) 391-400. doi:BBX42.

[26] HumphreysF.J., M. Hatherly, Recrystallization and Related Annealing Phenomena, Elsevier, 1995. doi:10.1016/B978-0-08-041884-1.50015-5.

[27] J.E. May, D. Turnbull, Secondary recrystallization in silicon iron, Trans. Am. Inst. Min. Metall. Eng. 212 (1958) 769-781.

[28] T. Gladman, On the Theory of the Effect of Precipitate Particles on Grain Growth in Metals, Proc. R. Soc. A Math. Phys. Eng. Sci. 294 (1966) 298-309. doi:10.1098/rspa.1966.0208.

[29] M.F. de Campos, Selected values for the stacking fault energy of face centered cubic metals, in: 6th Int. Lat. Am. Conf. Power Technol., Assoc Brasileira Ceram, Buzios, BRAZIL, 2007: pp. 708-711. doi:BIT18. 
[30] M. Soucail, M. Marty, H. Octor, Effect of high temperature deformation on grain growth in a PM nickel base superalloy, in: Superalloys 1996, 8th Int. Symp. superalloys proceedings (1996) 663-666.

[31] C.M. Heaney, M.L. Lasonde, A.M. Powell, B.J. Bond, C.M. O'Brien, Development of a New Cast and Wrought Alloy (René 65) for High Temperature Disk Applications, in: 8th Int. Symp. Superalloy 718 Deriv., 2014: pp. 67-77.

[32] M.-A. Charpagne, T. Billot, J.-M. Franchet, N. Bozzolo, Heteroepitaxial recrystallization: A new mechanism discovered in a polycrystalline $\gamma-\gamma^{\prime}$ nickel based superalloy, J. Alloys Compd. (2016). doi:10.1016/j.jallcom.2016.07.240.

[33] B.J. Bond, C.M. O’Brien, J.L. Russell, J.A. Heaney, M.L. Lasonde, René 65 Billet Material for Forged Turbine Components, in: U. John Wiley \& sons, Inc., Hoboken, NJ (Ed.), 8th Int. Symp. Superalloys718 Deriv., 2014: pp. 107-118. doi:10.1002/9781119016854.ch9.

[34] D. Prokoshkina, V.A. Esin, G. Wilde, S.V. Divinski, Grain boundary width, energy and selfdiffusion in nickel: Effect of material purity, Acta Mater. 61 (2013) 5188-5197. doi:10.1016/j.actamat.2013.05.010.

[35] M.-A. Charpagne, P. Vennegues, T. Billot, J.-M. Franchet, N. Bozzolo, Evidence of multimicrometric coherent $\gamma^{\prime}$ precipitates in a hot-forged $\gamma-\gamma^{\prime}$ nickel-based superalloy, J. Microsc. 263 (2016) 106-112. doi:10.1111/jmi.12380.

[36] M. Calcagnotto, D. Ponge, E. Demir, D. Raabe, Orientation gradients and geometrically necessary dislocations in ultrafine grained dual-phase steels studied by 2D and 3D EBSD, Mater. Sci. Eng. A. 527 (2010) 2738-2746. doi:10.1016/j.msea.2010.01.004.

[37] P.J. Konijnenberg, S. Zaefferer, D. Raabe, Assessment of geometrically necessary dislocation levels derived by 3D EBSD, Acta Mater. 99 (2015) 402-414. doi:10.1016/j.actamat.2015.06.051.

[38] G.D. Smith, H.L. Flower, Superplastic forming of Inconel Alloy 718SPF, in: ed. E. A. Loria (Ed.), Superalloys 718, 625, 706 Deriv., TMS, (1994) 355-64.

[39] M.-A. Charpagne, T. Billot, J.-M. Franchet, N. Bozzolo, Heteroepitaxial recrystallization observed in René 65 and Udimet 720: a new recrystallization mechanism possibly occuring also in other low lattice mismatch $\gamma / \gamma^{\prime}$ Nickel-based superalloys, in: Superalloys 2016, 13th Int. Symp. Superalloys proceedings (2016) 415-426. 
Graphical abstract
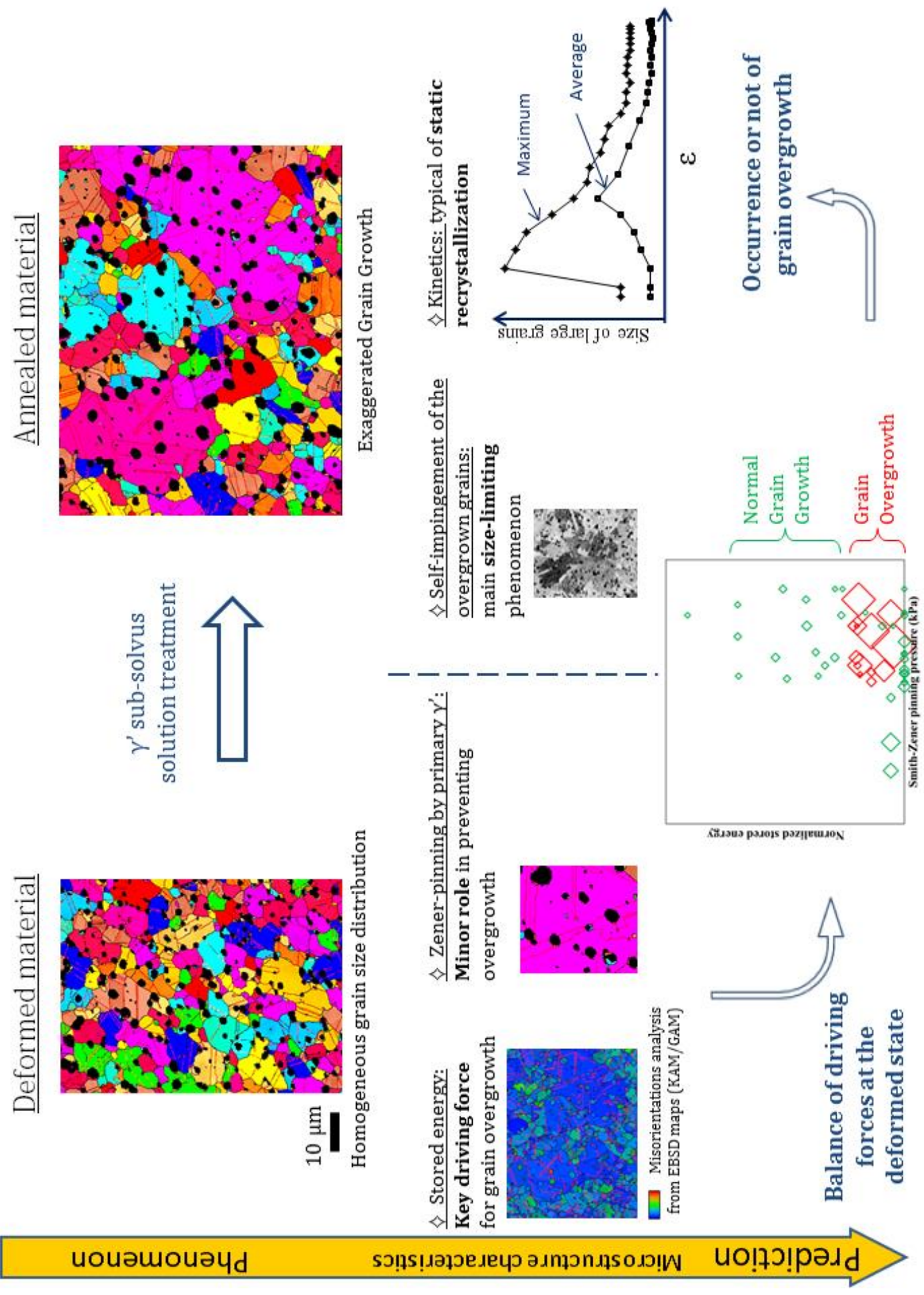